\title{
A CATEGORICAL GLIMPSE AT THE RECONSTRUCTION OF GEOMETRIES
}

\author{
Dedicated to Karl H. Hofmann, on the occasion of his 60 th birthday
}

\begin{abstract}
The author's reconstruction method ['Reconstruction of incidence geometries from groups of automorphisms', Arch. Math. 58 (1992) 621-624] is put in a categorical setting, and generalized to geometries with an arbitrary number of 'types'. The results amount to saying that the reconstruction process involves a pair of adjoint functors, and that the class of those geometries that are images under reconstruction forms a reflective subcategory.
\end{abstract}

The present paper deals with the problem whether a given geometry (i.e. roughly spoken, an incidence structure together with a group of automorphisms) is determined by the action of the group. After results on flagtransitive groups on incidence structures with two types of objects (see [7] for further historical notes), a first attempt by the author led to results that were quite satisfactory for the case of partial planes (see Proposition (2.7) below), and in particular for stable planes (see $[7,4]$ ). In a discussion about this previous result, K.H. Hofmann urged a categorical point of view. This categorical treatment led to new insights: some of the previous restrictions on the action of the group may be dropped, and the reconstruction process applies to geometries with an arbitrary number of types. Moreover, the categorical point of view helps the groups to their full rights: in fact, this paper presents an equivalence (in a strictly categorical sense) of 'sufficiently homogeneous' geometries and systems of subgroups.

\section{A CATEGORY OF INCIDENCE STRUCTURES}

(1.1) NOTATION. By Set and Gp we denote the familiar categories of sets and mappings, and of groups and group homomorphisms, respectively. The fact that a morphism (in any category) is monic (epic) shall be stressed by the notation $\rightarrow(\rightarrow)$. An isomorphism shall be denoted by $\Longrightarrow$, which is not to be confused with $\Rightarrow$ (logical implication). A natural number is defined as the set of its predecessors: $n:=\{m \mid m<n\}$. Thus 0 is the empty set, and $1=\{0\}$. For each mapping $f: X \rightarrow Y$ and each subset $U \subseteq Y$ we write $f^{\leftarrow}(U):=\{x \mid f(x) \in U\}$.

We shall consider categories that are not balanced, i.e. a morphism that is 
both monic and epic need not be an isomorphism. Hence the following classes of morphisms are of interest:

(1.2) DEFINITION. Consider any category.

(a) A monomorphism $m$ is called extreme, if for each factorization $m=h \circ g$ the fact that $g$ is epic implies that $g$ is an isomorphism:

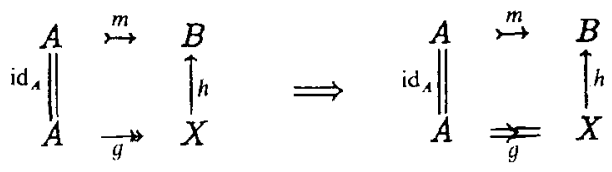

(b) Dually, an epimorphism $e$ is called extreme, if for each factorization $e=h \circ g$ the fact that $h$ is monic implies that $h$ is an isomorphism:

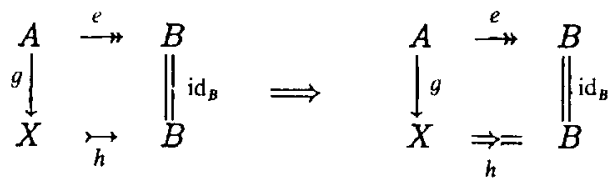

It is easy to see from the definition that an extreme monomorphism is an isomorphism if, and only if, it is an epimorphism; and dually. Note that Set and $\mathbf{G p}$ are balanced categories, where, in particular, each monic and each epic is extreme. We shall denote extreme monics and epics by the symbols $\hookrightarrow$ and $\rightarrow$, respectively.

(1.3) DEFINITION. An incidence structure $(I, A)$ consists of a family $A: T \rightarrow$ Set $: t \mapsto A_{t}$ and a subset $I \subseteq \Pi_{t \in T} A_{t}$. For incidence structures $(I, A)$ and $(J, B)$, a morphism $f=\left(F, f_{T}\right):(I, A) \rightarrow(J, B)$ consists of a mapping $f_{T}: T \rightarrow U$ (where $U$ is the domain of $B$ ), and a family $F: t \mapsto F_{t}$ with domain $T$ such that $F_{t}: A_{t} \rightarrow B_{f_{T}(t)}$ is a mapping (for each $t \in T$ ). Finally we require that there exists some mapping $F_{I}: I \rightarrow J$ such that

(A)

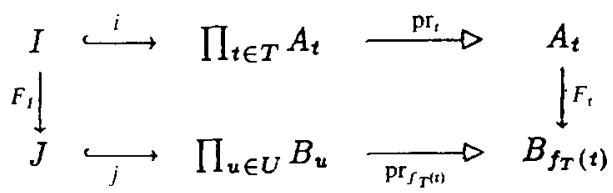

is commutative for each $t \in T$, where $i$ and $j$ denote the inclusion maps, and $\mathrm{pr}_{t}$ the projection. Every mapping $F_{I}$ that meets these requirements shall be called suited. These data define a category, which we shall denote by Inc. The 
following diagram makes clear how the composition $g \circ f$ of morphisms has to be defined (and that this definition works well):

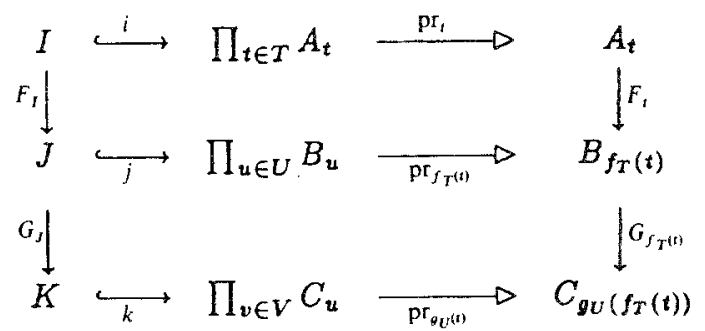

(1.4) INTERPRETATION. The domain $T$ of $A$ may be considered as set of types of geometric objects, while $I$ is an incidence relation in the spirit of $\mathrm{G}$. Pickert's definition* $[6: 1.1$, p. 2$]$. In this interpretation, a morphism is a mapping that preserves incidence and equality of type. Note that the 'type mapping' $f_{T}$ need not be monic nor epic; and that, in general, the mapping $F_{I}$ is not determined uniquely.

(1.5) LEMMA. Assume that $f=\left(F, f_{T}\right):(I, A) \rightarrow(J, B)$ is a morphism in Inc such that $f_{T}$ is bijective. Then the following holds:

(a) The suited mapping $F_{I}$ is determined uniquely by the family $F$.

(b) If $F_{t}$ is monic for each $t \in T$ then $F_{I}$ is monic.

Proof. If $f_{T}$ is a bijection then there exists the product mapping

$$
\varphi:=\prod_{t \in T}\left(F_{t} \circ \mathrm{pr}_{t}\right): \prod_{t \in T} A_{t} \rightarrow \prod_{t \in T} B_{f_{T^{(}(t)}}
$$

and the following diagram commutes for each suited $F_{I}$ :

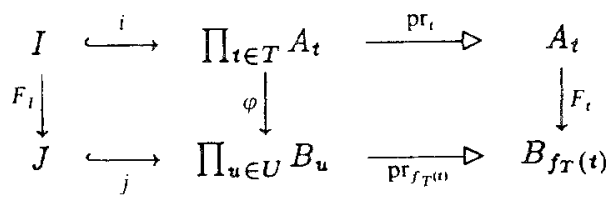

Since $j$ is monic, this implies that the suited mapping $F_{I}$ is unique. This proves assertion (a). If each of the $F_{t}$ is monic, then $\varphi$ is monic. Hence $F_{I}$ is monic, and assertion (b) is established. 
Note that a mapping with the properties of the product mapping (in particular, uniqueness!) need not exist if $f_{T}$ is not epic ${ }^{\star}$ or not monic.

(1.6) PROPOSITION. Let $f=\left(F, f_{T}\right):(I, A) \rightarrow(J, B)$ be a morphism in Inc.

(a) $f$ is epic if, and only if, $f_{T}$ and $F_{t}$ for each $t \in T$ (but not necessarily $F_{I}$ ) are epic.

(b) $f$ is monic if, and only if, $f_{T}$ and $F_{t}$ for each $t \in T$ are monic.

(c) An epimorphism $f$ is extreme if, and only if, every suited $F_{I}$ is epic.

(d) A monomorphism $f$ is extreme if, and only if $\star \star$,

$$
\left(\prod_{t \in T}\left(F_{t} \circ \mathrm{pr}_{t}\right)\right)^{\leftarrow}\left(\left(\prod_{t \in T} \operatorname{pr}_{f_{T}(t)}\right)(J)\right)=I .
$$

Proof. (i) Assume that $f_{T}$ is epic, and that $F_{t}$ is epic for each $t \in T$. We consider morphisms $g=\left(G, g_{U}\right):(J, B) \rightarrow(K, C)$ and $h=\left(H, h_{U}\right):(J, B) \rightarrow$ $(K, C)$ such that $g \circ f=h \circ f$. This means that $g_{U} \circ f_{T}=h_{U} \circ f_{T}$ and that $G_{f^{(t)}{ }^{\circ}} \circ F_{t}=H_{f_{T}(t)} \circ{ }^{\circ} F_{t}$ for each $t \in T$. The fact that $f_{T}$ and the $F_{t}$ are epimorphisms implies that $g_{U}=h_{U}$ and that $G_{u}=H_{u}$ for each $u \in U=f_{T}(T)$. Hence $f$ is epic.

(ii) Conversely, assume that either $f_{T}$ or one of the $F_{t}$ is not epic. We define a ('2-gon') incidence structure $(K, C)$ by $V:=2, C_{0}:=2=: C_{1}$, and $K:=2 \times 2$. Obviously, the mappings $g_{U} \equiv 0, G_{u} \equiv 0$ define a morphism $g=\left(G, g_{U}\right):(J, B) \rightarrow(K, C):$ e.g. $G_{J} \equiv(0,0)$ is suited. On the other hand, there exists a morphism $h=\left(H, h_{U}\right)$ such that

$$
\begin{aligned}
& h_{U}(u)= \begin{cases}0 & \text { if } u \in f_{T}(T) \\
1 & \text { otherwise }\end{cases} \\
& H_{u}(x)= \begin{cases}0 & \text { if } u=f_{T}(t) \text { and } x \in F_{t}\left(A_{t}\right) \\
1 & \text { otherwise. }\end{cases}
\end{aligned}
$$

Now $g \circ f=h \circ f$, but $g=h$ only if $f_{T}$ and each of the $F_{t}$ is epic. Thus assertion (a) is established.

(iii) Dualizing the arguments of (i), one obtains that $f$ is monic if $f_{T}$ and each of the $F_{t}$ are monic.

(iv) Assume that there are two types $s, t \in T$ such that $f_{T}(s)=f_{T}(t)$. We consider the incidence structure $(K, C)$ that is given by the settings $V:=1$,

*For example, consider the embedding of a line into 3-dimensional affine space: one is free to choose any plane that is incident with the line.

$\star \star$ If $f_{T}=\mathrm{id}_{T}$ this condition simplifies to the condition that $\left(\Pi_{t \in T} F_{t}\right)^{\leftarrow}(J)=I$. Note that the inclusion ' $\supseteq$ ' is already a consequence of the fact that $F_{I}$ is suited. 
$C_{0}:=0$, and $K:=0$. There are morphisms $g, h:(K, C) \rightarrow(I, A)$ such that $g_{K}(0)=s$ and $h_{K}(0)=t$. Now $f \circ g=f \circ h$, and we infer that $f$ is not monic. If, on the other hand, there is some $t \in T$ such that there are two elements $x, y \in A_{t}$ with $F_{t}(x)=F_{t}(y)$, then $V^{\prime}:=1, C_{0}^{\prime}:=1$, and $K^{\prime}:=0$ define an incidence structure $\left(K^{\prime}, C^{\prime}\right)$ that admits morphisms to $(I, A)$ that show that $f$ is not monic. Thus assertion (b) is established.

(v) In order to prove assertion (c), we fix a suited mapping $F_{I}$ and consider the incidence structure $\left(J^{\prime}, B\right)$ that is given by $J^{\prime}:=F_{I}(I)$. Obviously, the morphism $f$ may be regarded as a morphism from $(I, A)$ to $\left(J^{\prime}, B\right)$. Now the diagram

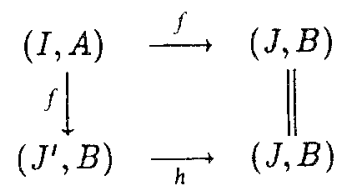

commutes, where $h_{U}:=\mathrm{id}_{U}$, and $H_{u}:=\mathrm{id}_{B_{u}}$ for each $u \in U$ (the inclusion $H_{J^{\prime}}: J^{\prime} \rightarrow J$ is suited). If $f$ is an extreme epimorphism, we conclude that $h$ is an isomorphism, hence $J^{\prime}=J$.

(vi) Assume that $f$ is an epimorphism and that every suited $F_{I}$ is epic. If $f=h \circ g$ for morphisms $g:(I, A) \rightarrow(K, C)$ and $h:(K, C) \rightarrow(J, B)$ and $h$ is monic, then $h$ is epic (since $f$ is), and we infer that $h_{K}$ and each of the $H_{v}$ are bijections. Since $F_{I}:=H_{K} \circ G_{J}$ is suited, it is epic, and this holds for $H_{K}$, too. Thus $H_{K}$ is a bijection by Lemma (1.5(b)), and $h$ is an isomorphism. This proves assertion (c).

(vii) For the proof of assertion (d) we introduce the ad hoc notation

$$
\begin{aligned}
\pi & :=\prod_{t \in T} \operatorname{pr}_{F_{T}(t)}: \prod_{u \in U} B_{u} \rightarrow \prod_{t \in T} B_{f_{T}(t)} \\
\varphi & :=\prod_{t \in T}\left(F_{t} \circ \operatorname{pr}_{t}\right): \prod_{t \in T} A_{t} \rightarrow \prod_{t \in T} B_{f_{T}(t)} \\
I^{\prime} & :=\varphi^{\leftarrow}(\pi(J))=\left(\prod_{t \in T}\left(F_{t} \circ \operatorname{pr}_{t}\right)\right)^{\leftarrow}\left(\left(\prod_{t \in T} \operatorname{pr}_{f_{T}(t)}\right)(J)\right)
\end{aligned}
$$

We obtain an incidence structure $\left(I^{\prime}, A\right)$. From the commutativity of $(\mathbf{A})$ we infer that $I \subseteq I^{\prime}$. Now for each $x \in I^{\prime}$, there exists some $y_{x} \in J$ such that $\varphi(x)=\pi\left(y_{x}\right)$. Any choice of such a $y_{x}$ for each $x \in I^{\prime}$ defines a suited mapping $F_{I^{\prime}}: x \mapsto y_{x}$. Hence we have the following commuting diagram: 


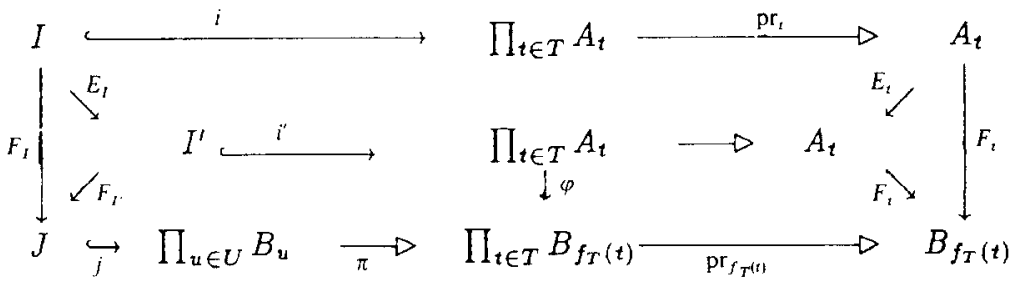

Here for each $t \in T$, the identity $\mathrm{id}_{A_{t}}$ is denoted by $E_{t}$, and $E_{I}: I \hookrightarrow I^{\prime}$ is the inclusion.

(viii) If $f$ is an extreme monic, we conclude that $\left(E\right.$, id $\left._{T}\right):(I, A) \rightarrow\left(I^{\prime}, A\right)$ is an isomorphism. This implies that $E_{I}$ is a bijection (cf. (1.5)), hence $I^{\prime}=I$.

(ix) Finally, assume that $I=I^{\prime}$, and that $f$ is monic. Let $g=\left(G, g_{T}\right):(I, A) \rightarrow(K, C)$ and $h=\left(H, h_{V}\right):(K, C) \rightarrow(J, B)$ be morphisms such that $g$ is epic and $f=h \circ g$. Then $g$ is monic (since $f$ is), hence $g_{T}$ and each of the $G_{t}$ are bijections. According to (1.5) there is a unique suited mapping $G_{I}$, and $G_{I}$ is monic. We have the following diagram:
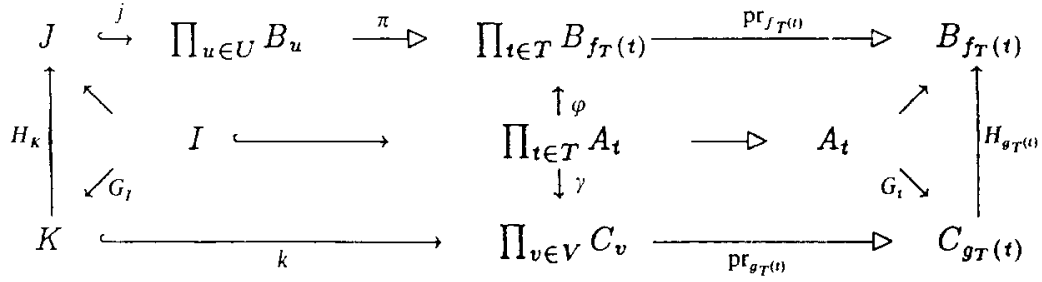

Here $\gamma:=\Pi_{t \in T} G_{t}^{\circ} \mathrm{pr}_{t}$. From the relation

$$
\varphi^{\leftarrow}\left(\pi\left(H_{K}(K)\right)\right) \subseteq \varphi^{\leftarrow}(\pi(J))=I^{\prime}
$$

we infer that

$$
K \subseteq \gamma\left(\varphi^{\leftarrow}\left(\pi\left(H_{K}(K)\right)\right)\right) \subseteq \gamma\left(I^{\prime}\right) .
$$

Since $\gamma\left(I^{\prime}\right)=\gamma(I)=G_{I}(I)$ and $k$ is monic, we conclude that $G_{I}$ is epic. Hence $g$ is an isomorphism, and assertion (d) is proved.

Later on, we shall be interested mainly in morphisms $\left(F, f_{T}\right)$ that preserve types (in the sense that $f_{T}=\mathrm{id}_{T}$, or at least is a bijection). In this case, extreme epimorphisms are easier to recognize:

(1.7) LEMMA. Assume that $f=\left(F, f_{T}\right):(I, A) \rightarrow(J, B)$ is an epimorphism in Inc, and that $f_{T}$ is a bijection. Then fis extreme if, and only if, there exists a suited $F_{I}$ that is epic.

Proof. This follows immediately from (1.5(a)) and (1.6(c)). 
(1.8) REMARK. Similar arguments may be used to show that extreme monics (epics) in Inc are embeddings (identifications) ${ }^{\star}$.

(1.9) EXAMPLES. (a) In descriptive geometry, one is particularly interested in extreme monomorphisms: e.g. a drawing of a polyhedron may be interpreted as a morphism from a (finite) incidence structure to the real affine plane. Of course one tries to avoid that the drawing "shows' incidences that do not occur in the original object.

(b) Closure theorems give examples of situations where one is interested in monomorphisms that are not extreme. For example, the validity of Desargues' theorem may be phrased in the following way: Let $(I, A)$ be the incidence structure that is obtained by 'drawing two perspective triangles, the three points where corresponding sides meet, and a line that contains exactly two of these three points'. Then Desargues' theorem is valid in $(J, B)$ if there is no monomorphism from $(I, A)$ to $(J, B)$ that is extreme.

(c) Examples of morphisms that change types are given by dualities. Further examples are the embeddings of rank 2 residues in diagram geometries, cf. [3].

\section{GeOMETRIES, SKETCHES AND PICTURES}

We turn to geometries in a sense inspired by F. Klein:

(2.1) DEFINITION. Assume that $(I, A)$ is an incidence structure, and let $\Gamma$ be a group. A geometry $(\gamma, I, A)$ is given by a family $\gamma$ (with domain $T$ ) of group actions $\gamma_{t}: \Gamma \times A_{t} \rightarrow A_{t}$ such that there exists an action $\gamma_{I}: \Gamma \times I \rightarrow I$ making the following diagram commutative:

(B)

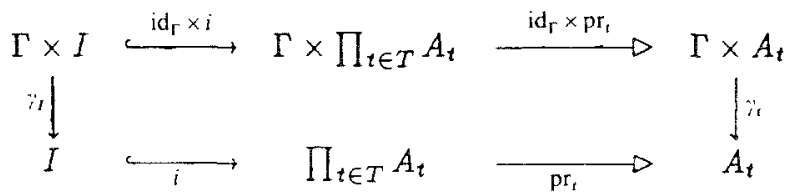

Note that $\gamma_{I}$ is uniquely determined, cf. (1.5).

A morphism $(\varphi, f)=\left(\varphi, F, f_{T}\right):(\gamma, I, A) \rightarrow(\delta, J, B)$ consists of a morphism $\varphi: \Gamma \rightarrow \Delta$ (in Gp) and a morphism $f=\left(F, f_{T}\right):(I, A) \rightarrow(J, B)$ such that commutes for some suited $F_{I}$. These data define a category, which we shall denote by Geo. There are the forgetful functors

$\mathbf{U}_{\text {Inc }}: \mathbf{G e o} \rightarrow$ Inc: $(\gamma, I, A) \mapsto(I, A)$ and $\mathbf{U}_{\mathbf{G p}}: \mathbf{G e o} \rightarrow \mathbf{G p}:(\gamma, I, A) \mapsto \Gamma$.

*Here we use the terms 'embedding' and 'identification' in the sense of 'Einbettung' and 'Identifizierung', as defined in [2, 4.8.1, 4.10]. 

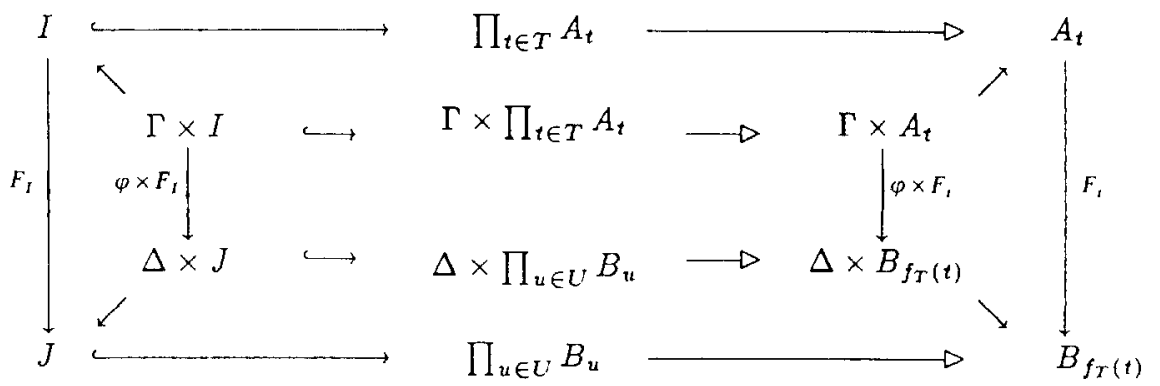

(2.2) PROPOSITION. There are initial and terminal objects (but no null objects ${ }^{\star}$ ) in the categories Inc and Geo:

(i) There exists exactly one object of Inc with no types at all; in this case, the family $A$ (and hence also $I$ ) is empty. The corresponding incidence structure $(0,0)$ is initial in Inc. Fixing the (cardinality of the) set $T$ of

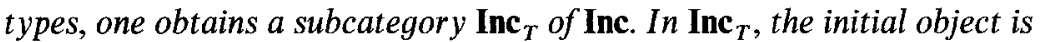
given by $\left(0,(0)_{t \in T}\right)$, where $(0)_{t \in T}: T \rightarrow$ Set: $t \mapsto 0$.

(ii) The (unique) action of the initial $1 \in \mathbf{G p}$ on the empty set gives rise to the initials $\mathfrak{I}$ and $\mathfrak{I}_{T}$ in $\mathbf{G e o}$ and $\mathbf{G e o}_{T}$, respectively, where $\mathbf{U}_{\mathbf{I n c}}(\mathfrak{I})=(0,0)$ and $\mathbf{U}_{\text {Inc }}\left(\mathfrak{I}_{T}\right)=\left(0,(0)_{t \in T}\right)$.

(t) The setting $T=A_{0}=I=1$ defines a terminal object $(1,(1)) \in \mathbf{I n c}$, while $I=1$ and $A \equiv 1$ gives the terminal object $\left(1^{T},(1)_{t \in T}\right) \in \mathbf{I n c}_{T}$.

(tt) The (unique) action of 1 on 1 gives terminals $\mathfrak{I}$ and $\mathfrak{I}_{T}$ in $\mathbf{G e o}$ and $\mathbf{G e o}_{T}$, respectively, where $\mathbf{U}_{\text {Ind }}(\mathfrak{I})=(1,(1))$ and $\mathbf{U}_{\text {Inc }}\left(\left(\mathfrak{I}_{T}\right)\right)=\left(1^{T},(1)_{t \in T}\right)$.

The proof of these statements is straightforward and left to the reader.

The initial $1 \in \mathbf{G p}$ acts on each incidence structure $(K, C)$ in a unique way; we shall denote this action by $(v, K, C)$. In particular, we obtain that the functor $\mathbf{U}_{\text {Inc }}$ is full. (This is in contrast to familiar forgetful functors, e.g. $\mathbf{G p} \rightarrow$ Set.) Note that for each group $\Psi$ there are unique actions $\Psi \times 0 \rightarrow 0$ and $\Psi \times 1 \rightarrow 1$. This implies that there are families $\imath$ and $\tau$ of actions such that $(\imath, 0,0)$ and $(\tau, 1,(1))$ are geometries. We shall use these objects in the proof of the following statement.

(2.3) LEMMA. The functors $\mathbf{U}_{\mathbf{I n c}}$ and $\mathbf{U}_{\mathbf{G p}}$ preserve monics and epics.

Proof. (i) Assume that $f$ is not epic. Then there are morphisms $g, h:(J, B) \rightarrow(K, C)$ such that $g \circ f=h \circ f$ but $g \neq h$. Now the unique morphism $\omega:(\delta, J, B) \rightarrow \mathfrak{T}$ factors through morphisms $(\alpha, g)$ and $(\beta, h)$ from $(\delta, J, B)$ to

$\star$ If one requires the sets $I, T$, and $A_{t}$ to be non-empty, then $(1,(1))$ and $\left(1^{T},(1)_{\tau \in T}\right)$ are null objects in Inc and $\mathbf{I n c}_{T}$, respectively; while $\mathfrak{T}$ and $\mathfrak{I}_{T}$ are null objects in $\mathbf{G e o}$ and $\mathbf{G e o}_{T}$, respectively. 
$(v, K, C)$. Now $\alpha \neq \beta$ implies that $(\alpha, g) \neq(\beta, h)$, but $(\alpha, g) \circ(\varphi, f)=$ $(\beta, h) \circ(\varphi, f)$, hence $(\varphi, f)$ is not epic. This shows that $\mathrm{U}_{\text {Ine }}$ preserves epics. Dual arguments (with $\mathfrak{I}$ instead of $\mathfrak{I}$ ) show that $\mathbf{U}_{\text {Inc }}$ preserves monics.

(ii) Assume that $\varphi$ is not epic. Then there are morphisms $\alpha, \beta: \Delta \rightarrow \Psi$ such that $\alpha \circ \varphi=\beta \circ \varphi$ but $\alpha \neq \beta$. Now the unique morphism $\omega:(\delta, J, B) \rightarrow \mathfrak{I}$ factors through morphisms $(\alpha, g)$ and $(\beta, h)$ from $(\delta, J, B)$ to $(\tau, 1,(1))$. Now $\alpha \neq \beta$ implies that $(\alpha, g) \neq(\beta, h)$, but $(\alpha, g) \circ(\varphi, f)=(\beta, h) \circ(\varphi, f)$, hence $(\varphi, f)$ is not epic. This shows that $\mathbf{U}_{\mathbf{G p}}$ preserves epics. Dual arguments (with $(l, 0,0)$ instead of $(\tau, 1,(1)))$ show that $\mathbf{U}_{\mathbf{G p}}$ preserves monics.

Note, however, that neither $\mathbf{U}_{\mathbf{I n c}}$ nor $\mathbf{U}_{\mathbf{G p}}$ reflects monics or epics.

(2.4) PROPOSITION. Let $(\varphi, f):(\gamma, I, A) \rightarrow(\delta, J, B)$ be a morphism in Geo.

(a) $(\varphi, f)$ is epic if, and only if, $\varphi$ and $f$ are epic.

(b) $(\varphi, f)$ is monic if, and only if, $\varphi$ and $f$ are monic.

(c) An epimorphism $(\varphi, f)$ is extreme if, and only if, $f$ is extreme.

(d) A monomorphism $(\varphi, f)$ is extreme if, and only if, $f$ is extreme.

Proof. According to (2.3), the fact that $(\varphi, f)$ is epic (monic) implies that both $\varphi$ and $f$ are epic (monic). Since the composition of morphisms in Geo is defined by $(\beta, h) \circ(\alpha, g):=(\beta \circ \alpha, h \circ g)$, the product functor $\mathbf{U}_{\mathbf{G p}} \times \mathbf{U}_{\mathbf{I n c}}$ reflects monics and epics. Thus assertions (a) and (b) are proved, and (c), (d) follow.

Given a geometry $\mathfrak{5}=(\gamma, I, A)$, one may try to recover information about $\mathfrak{b}$ from the family $\gamma$ of actions. Of course, this attempt will yield interesting results only if this family is 'sufficiently strong'. Our next definition is a precision of 'strength':

(2.5) DEFINITIONS. (a) Let $\mathfrak{G}=(\gamma, I, A)$ be a geometry. A family $L: T \rightarrow$ Set is said to sketch $\mathfrak{G}$, if the following conditions hold:

- For each $t \in T$, the set $L_{t}$ is a set of representatives for the orbit decomposition of $A_{t}$ under the action $\gamma_{t}$.

- The product $L_{I}:=\Pi_{t \in T} L_{t}$ is contained in $I$, and forms a set of representatives for the orbit decomposition of $I$ under the action $\gamma_{I}$.

If $L$ sketches (5, then $(L, \gamma, I, A)$ is called a sketched geometry. A morphism

$$
(\varphi, f):(L, \gamma, I, A) \rightarrow(M, \delta, J, B)
$$

of sketched geometries is a morphism $(\varphi, f):(\gamma, I, A) \rightarrow(\delta, J, B)$ in Geo such that $F_{t}\left(L_{t}\right) \subseteq M_{t}$ for each $t \in T$ (then $F_{Y}\left(L_{I}\right) \subseteq M_{J}$ for each suited $\left.F_{I}\right)$. Thus we obtain a category SGeo.

(b) Let $\Gamma$ be a group, and denote the set of all subgroups of $\Gamma$ by $\sigma(\Gamma)$. A 
sketch $(\Gamma, \mathscr{L})$ is given by a family $\mathscr{L}: T \rightarrow 2^{\sigma(\Gamma)}: t \mapsto \mathscr{L}_{t}$ (i.e. $\mathscr{L}_{t}$ is a set of subgroups of $\Gamma)$. A morphism $\left(\varphi, \Phi, f_{T}\right):(\Gamma, \mathscr{L}) \rightarrow(\Delta, \mathscr{M})$ consists of a morphism $\varphi: \Gamma \rightarrow \Delta$ (in $\mathbf{G p}$ ), a mapping $f_{T}: T \rightarrow U$ (where $U$ is the domain of $\mathscr{M}$ ), and a family $\Phi: t \mapsto \Phi_{t}$ with domain $T$ such that

- $\Phi_{t}: \mathscr{L}_{t} \rightarrow \mathscr{M}_{f_{T(t)}}$ is a mapping for each $t \in T$.

- For each $\Lambda \in \mathscr{L}_{t}$, the inclusion $\varphi(\Lambda) \leqslant \Phi_{t}(\Lambda)$ holds.

These data define a category Sk of sketches.

(2.6) PROPOSITION. The family $Z$, where $Z_{t}=\{1\}$ for each $t$, sketches the terminal $\mathfrak{T}$ resp. $\mathfrak{I}_{T}$. Thus one obtains null objects in $\mathbf{S G e o \text { . }}$

Again, the (straightforward) proof is left to the reader.

The existence of a family $L$ that sketches a given geometry $(\gamma, I, A)$ is rather a strong condition:

(2.7) PROPOSITION. Let $(I, A)$ be a partial plane ${ }^{\star}$ with point space $A_{p}$ and line space $A_{l}$, and assume that there are families $\gamma$ and $L$ such that $(L, \gamma, I, A) \in \mathbf{S G e o}$. Then either $L_{p}$ or $L_{l}$ has exactly one element.

Proof. Assume that there are points $x, y \in L_{p}$ and lines $v, w \in L_{l}$. Then

$$
\{(x, v),(x, w),(y, v),(y, w)\} \subseteq I,
$$

hence either $x=y$ or $v=w$ by the definition of a partial plane.

In particular, this means that each sketched finite linear space is point homogeneous, cf. [1, Th. 2.1]. Outside the realm of partial planes, one can easily cook up examples with non-trivial sets $L_{t}$ :

(2.8) EXAMPLE. Assume that the points $p_{n}(1 \leqslant n \leqslant 4)$ form the vertices of a tetrahedron, and denote the sides of this tetrahedron by $s_{n}$ in such a way that $p_{n}$ lies on $s_{m}$ if $n \neq m$. The transpositions (12) and (34) generate a subgroup $\Gamma$ of $\mathrm{Sym}_{4}$ that acts in the obvious way on the vertices and on the sides of the tetrahedron. Now the sets $L_{p}:=\left\{p_{1}, p_{3}\right\}$ and $L_{s}:=\left\{s_{2}, s_{4}\right\}$ sketch the given geometry.

There is a rather obvious way to associate a sketch with each sketched geometry $\left(\mathfrak{s}=(L, \gamma, I, A)\right.$, namely to define $\mathscr{L}_{t}:=\left\{\Gamma_{r}^{t} \mid r \in L_{t}\right\}$ where $\Gamma_{r}^{t}$ denotes the stabilizer of $r$ with respect to the action $\gamma_{t}$. Then $S((5)):=(\Gamma, \mathscr{L})$ is a sketch. For the reversed direction, from $\mathbf{S k}$ to $\mathbf{S G e o ,}$ we have to work a little harder:

${ }^{\star}$ In the sense of M. Hall [4]: i.e. there are two types ('points' and 'lines'): $T=\{p, l\}$, and an incidence relation $I \subseteq A_{p} \times A_{l}$ such that for any two points $x, y \in A_{p}$ there is at most one line $w \in A_{l}$ with $\{(x, w),(y, w)\} \subseteq I$, and dually. 
(2.9) DEFINITION. Let $\Xi=(\Gamma, \mathscr{L})$ be a sketch. For each $t \in T$, write

$$
P_{t}:=P_{t}(\Im):=\bigcup_{\Lambda \in \mathscr{L}_{t}} \frac{\Gamma}{\Lambda},
$$

and let $\lambda_{t}$ be the action of $\Gamma$ on $P_{t}$ via left multiplication. With

$$
P_{I}:=P_{I}(\Im):=\left\{\left(\alpha \Lambda_{t}\right)_{t \in T} \mid \alpha \in \Gamma, \Lambda_{t} \in \mathscr{L}_{t}\right\},
$$

and the action $\lambda_{I}$ of $\Gamma$ on $P_{I}$ via (simultaneous) left multiplication, we obtain a geometry $\left(\lambda, P_{I}, P\right)$ :

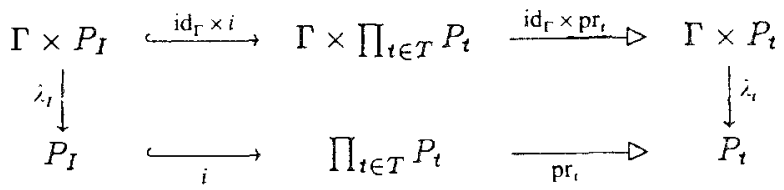

Obviously, the family $\mathscr{L}$ sketches $\left(\lambda, P_{I}, P\right)$. (Note that $P_{I}$ is just the union of the orbits of the elements of $\Pi_{t \in T} \mathscr{L}_{t}$ under the action $\lambda_{I}$.) We shall call $\left(\mathscr{L}, \lambda, P_{I}, P\right)$ the picture of $(\Gamma, \mathscr{L})$ and denote it by $\mathbf{P}(\Gamma, \mathscr{L})$.

\section{RECONSTRUCTION}

The categorical view of the reconstruction of sketched geometries amounts to the proof that the reconstruction process involves a pair of adjoint functors (with object mappings $\mathbf{S}$ and $\mathbf{P}$, respectively), and that the class of those geometries that are images under reconstruction forms a reflective subcategory.

Straightforward verification shows:

(3.1) LEMMA. For each morphism $\left(\varphi, F, f_{T}\right):(L, \gamma, I, A) \rightarrow(M, \delta, J, B)$ define

$$
\mathbf{S}\left(\varphi, F, f_{T}\right):=\left(\varphi, \Phi, f_{T}\right)
$$

where $\Phi_{t}$ is given by $\Phi_{t}\left(\Gamma_{r}^{t}\right):=\Delta_{F_{t}(r)}^{f_{r}(t)}$. Then $\mathbf{S}: \mathbf{S G e o} \rightarrow \mathbf{S k}$ is a functor.

A first step towards the understanding of the connection between $\mathbf{S}$ and $\mathbf{P}$ is the following trivial observation:

(3.2) LEMMA. $\mathbf{S} \circ \mathbf{P}=\mathrm{id}_{\mathbf{S k}}$.

The study of $\mathbf{P} \circ \mathbf{S}$ requires some more effort.

(3.3) THEOREM. For each sketched geometry $(\tilde{\mathfrak{n}}=(L, \gamma, I, A)$, there is an extreme epimorphism $\eta_{\mathfrak{G}}=\left(\mathrm{id}_{\Gamma}, \mathrm{H}, \mathrm{id}_{T}\right):\left(\mathfrak{5} \rightarrow \mathbf{P}(\mathbf{S}(\mathfrak{G}))\right.$. The epimorphism $\eta_{\mathfrak{G}}$ is 
an isomorphism if, and only if, the following holds: If $r, s \in L_{t}$, the equality $\Gamma_{r}^{t}=\Gamma_{s}^{t}$ implies that $r=s$.

Proof. For each $x \in A_{t}$, there is a (unique) representative $r \in L_{t}$ and a (usually not unique) element $\alpha \in \Gamma$ such that $x=\gamma_{t}(\alpha, r)$. If $\gamma_{t}(\alpha, r)=\gamma_{t}(\beta, s)$ for some $(\beta, s) \in \Gamma \times L_{t}$, then $\alpha^{-1} \beta \in \Gamma_{r}^{t}$ and $r=s$. This implies that the mapping

$$
\mathbf{H}_{t}: A_{t} \rightarrow P_{t}: \gamma_{t}(\alpha, r) \mapsto \alpha \Gamma_{r}^{t}
$$

is well defined, and that it is injective if, and only if, for any two representatives $r, s \in L_{t}$ the stabilizers $\Gamma_{r}^{t}$ and $\Gamma_{s}^{t}$ are different. (Note, however, that for different types $t, u \in T$, there may hold an equation $\Gamma_{r}^{t}=\Gamma_{s}^{u}$ without disturbing the injectivity of any of the $\mathrm{H}_{t}$, cf. Example (2.8).) Similar arguments show that

$$
\mathrm{H}_{I}: I \rightarrow P_{I}: \gamma_{I}\left(\alpha,\left(r_{t}\right)_{t \in T}\right) \mapsto\left(\alpha \Gamma_{r_{t}}^{t}\right)_{t \in T}
$$

is a well-defined mapping. Obviously, the family $\mathrm{H}$ defines an extreme epimorphism $\eta_{\mathfrak{G}}=\left(\mathrm{id}_{\Gamma}, \mathrm{H}, \mathrm{id}_{T}\right)$. The assertion that $\eta_{\mathfrak{G}}$ is an isomorphism is equivalent to the assertion that $\eta_{\mathfrak{G}}$ is monic, hence equivalent to the assertion that for each $t \in T$ the mapping $\mathrm{H}_{t}$ is monic.

(3.4) THEOREM. $\langle\mathbf{S}, \mathbf{P}, \psi\rangle: \mathbf{S G e o} \rightarrow \mathbf{S k}$ is an adjunction with unit $\eta=\left(\left(\mathfrak{5} \mapsto \eta_{\mathfrak{5}}\right)\right.$ and counit $\varepsilon=\left(\varsigma_{\mapsto} \mapsto \mathrm{id}_{\mathscr{G}}\right)$, where

$\left(\psi_{\mathfrak{G}, \Subset}\right)^{-1}: \mathbf{S G e o}(\mathfrak{G}, \mathbf{P \subseteq}) \rightarrow \mathbf{S k}\left(\mathbf{S}\left(\mathfrak{5}, \Xi_{)}\right):\left(\varphi, F, f_{T}\right) \mapsto \mathbf{S}\left(\varphi, F, f_{T}\right)\right.$.

In particular, we have that $\mathbf{P}: \mathbf{S k} \rightarrow \mathbf{S G e o}$ is a functor, and that $\eta$ is a natural transformation from $\mathrm{id}_{\mathbf{S G e o}_{\mathrm{G}}}$ to $\mathbf{P} \circ \mathbf{S}$.

Proof. Since $\varepsilon_{(\Gamma, \mathscr{L})}=\mathrm{id}_{(\Gamma, \mathscr{L})}$ is a universal arrow from $\mathrm{S}$ to $(\Gamma, L)$, this follows from [5, Th. IV.1.2(iv)].

Since each component $\varepsilon_{\subseteq}$ of the counit $\varepsilon$ is an isomorphism, we obtain [5, Th. IV.3.1]:

(3.5) COROLLARY. The functor $\mathbf{P}$ is full and faithful.

(3.6) THEOREM. The image of $\mathbf{S k}$ under $\mathbf{P}$ in $\mathbf{S G e o}$ forms a reflective subcategory.

Proof. Using (3.2) we obtain that $\mathbf{P} \circ \mathbf{S} \circ \mathbf{P}=\mathbf{P}$, hence $\left.\mathbf{P} \circ \mathbf{S}\right|_{\mathbf{P}(\mathbf{S k})}=\mathrm{id}_{\mathbf{P}(\mathbf{S k})}$. Now [5, Th. IV.1.2(v)] says that $\mathbf{P} \circ \mathbf{S}$ is a left adjoint for the inclusion functor.

Quite often, one wishes to identify a certain object in an incidence structure with the set of objects that are incident with it. Generalizing notions like 'point row' or 'line pencil', we introduce residues in an incidence structure $(I, A)$ with type set $T$ by the following procedure: Fix an element $r$ of type $u$, 
consider all objects that are 'in some flag together with $r$ ', and introduce an incidence structure with type set $T \backslash\{u\}$ on these objects. To be formal:

(3.7) DEFINITION. Let $(I, A)$ be an incidence structure, and fix some type $u \in T$ and an element $r \in A_{u}$. Writing $\hat{A}_{t}:=\operatorname{pr}_{t}\left(I \cap \operatorname{pr}_{u}^{-}(r)\right)$ for each $t \in T \backslash\{u\}$ defines a family $\hat{A}$ of sets with domain $T \backslash\{u\}$. With

$$
\hat{I}:=\left\{\left(\operatorname{pr}_{t}(x)\right)_{t \in T \backslash\{u\}} \mid x \in I \cap \operatorname{pr}_{u}^{\leftarrow}(r)\right\} \subseteq \prod_{t \in T \backslash\{u\}} \hat{A}_{t}
$$

we obtain an incidence structure $(\hat{I}, \hat{A})$. To stress the choice of $u \in T$ and $r \in A_{u}$, we write $\operatorname{Res}_{r}^{u}:=(\hat{I}, \hat{A})$.

(3.8) PROPOSITION. Assume that $(L, \gamma, I, A)$ is a sketched geometry, and fix $r \in L_{u}$ for some type $u \in T$. Then $(\hat{L}, \hat{\gamma}, \hat{I}, \hat{A})$ is a sketched geometry, where $(\hat{I}, \hat{A})=\operatorname{Res}_{\mathrm{r}}^{u}$ as in $(3.7)$, and

$$
\hat{\gamma}_{t}:=\left.\gamma_{t}\right|_{r} ^{u} \times \hat{A}_{t}, \quad \hat{L}:=L \mid \Gamma \backslash\{u\} .
$$

Proof. By definition of $\Gamma_{r}^{u}$, we have that $\hat{\gamma}_{t}\left(\Gamma_{r}^{u} \times \hat{A}_{t}\right) \subseteq \hat{A}_{t}$ for each $t \in T \backslash\{u\}$. This implies that $(\hat{\gamma}, \hat{I}, \hat{A})$ is a geometry. In order to show that $\hat{L}$ sketches $(\hat{\gamma}, \hat{I}, \hat{A})$, we observe that each element $x \in I$ is of the form $x=\gamma_{I}\left(\alpha,\left(r_{t}\right)_{t \in T}\right)=\left(\gamma_{t}\left(\alpha, r_{t}\right)\right)_{t \in T}$ for some $\alpha \in \Gamma$ and unique representatives $r_{t} \in L_{t}$. Now $\gamma_{u}\left(r_{u}\right)=r$ implies that $r_{u}=r$, hence $\alpha \in \Gamma_{r}^{u}$ if, and only if, the element $x$ belongs to $I \cap \mathrm{pr}_{u}^{\leftarrow}(r)$. This yields that for each $t \in T \backslash\{u\}$, the set $\hat{L}_{t}$ is a set of representatives for the action $\hat{\gamma}_{t}$ of $\Gamma_{r}^{u}$ on $\hat{A}_{t}$, and that $\Pi_{t \in T \backslash\{u\}} \hat{L}_{i}$ forms a set of representatives for the action

$$
\hat{\gamma}_{\hat{I}}=\left(\left(\alpha,\left(x_{t}\right)_{t \in T \backslash\{u\}}\right) \mapsto\left(\hat{\gamma}_{t}\left(\alpha, x_{t}\right)\right)_{t \in T \backslash\{u\}}\right)
$$

(3.9) COROLLARY. Assume that $\mathbf{5}=(L, \gamma, I, A)$ is a sketched geometry. Then for each type $u \in T$, the equality $\Gamma_{r}^{u}=\Gamma_{t}^{u}$ for $r, t \in L_{u}$ implies that $\operatorname{Res}_{r}^{u}=\operatorname{Res}_{t}^{u}$.

Proof. This follows easily from the fact that, for each $t \in T$, there holds the equation

$$
\operatorname{pr}_{t}\left(I \cap \operatorname{pr}_{u}^{-}(r)\right)=\gamma_{t}\left(\Gamma_{r}^{u} \times L_{t}\right)
$$

In view of (3.9), it seems reasonable to consider the full subcategory RGeo of SGeo, consisting of those sketched geometries $(L, \gamma, I, A)$ such that the following condition holds:

(R) For every $t \in T$ and $r, s \in L_{t}$, the equality $\operatorname{Res}_{r}^{t}=\operatorname{Res}_{s}^{t}$ implies that $r=s$.

In fact, it is easy to find examples that show that condition (R) does not imply that for any two objects $x, y \in A_{t}$ we have the implication 
$\operatorname{Res}_{x}^{t}=\operatorname{Res}_{y}^{t} \Rightarrow x=y$. Though it seems somewhat arbitrarily, condition (R) singles out an important subcategory:

(3.10) THEOREM. The categories RGeo and Sk are equivalent (via $\eta$ ).

Proof. From (R) we infer that for $r, s \in L_{t}$, the equation $\Gamma_{r}^{t}=\Gamma_{s}^{t}$ implies that $r=s$. According to (3.3), this means that $\eta_{\mathfrak{G}}$ is an isomorphism for each object (j) of RGeo.

(3.11) FINAL REMARK. In our present treatment, the situation that was studied in [7] occurs as a special case, namely: The geometries under consideration have two types ('points' and 'lines'), and are subject to three conditions (R1), (R2), and (R3). Condition (R2) says that one has a sketched geometry, with the additional property $(R 1)$ that the actions on the points is transitive (i.e. a property that is quite natural in the realm of linear spaces, of. (2.7)). Condition (R3) says that stabilizers of different representatives are different. Thus it makes sure that the reconstruction process yields an isomorphism. Our result (3.10) above implies that (R3) in [7] may be replaced by condition ( $R$ ), without affecting the results of [7]. In particular, the validity of (R3) in the examples [7,7] holds a fortiori.

\title{
REFERENCES
}

1. Block, R. E., 'On the orbits of collineation groups', Math. Z. 96 (1967), 33-49.

2. Brinkmann, H.-B. and Puppe, D., Kategorien und Funktoren, Springer, 1966.

3. Buekenhout, F., 'Diagrams for geometries and groups', J. Combin. Theory, Ser. A 27 (1979), 121-151.

4. Hall, M., 'Projective planes', Trans. Amer. Math. Soc. 54 (1943), 229-277.

5. MacLane, S., Categories for the Working Mathematician, Springer, 1971.

6. Pickert, G., Projektive Ebenen, Springer, 1955.

7. Stroppel, M., 'Reconstruction of incidence geometries from groups of automorphisms', Arch. Math. 58 (1992) 621-624.

\author{
Author's address: \\ Markus Stroppel, \\ Fachbereich Mathematik, \\ Technische Hochschule Darmstadt, \\ Schloßgartenstr. 7, \\ D-6100 Darmstadt, \\ Germany.
}

(Received, March 18, 1992; revised version, June 15, 1992) 